\title{
Aldo-keto reductases are biomarkers of NRF2 activity and are co-ordinately overexpressed in non-small cell lung cancer
}

\author{
A Kenneth MacLeod ${ }^{1}$, Lourdes Acosta-Jimenez ${ }^{1}$, Philip J Coates ${ }^{1,4}$, Michael McMahon ${ }^{1}$, Frank A Carey ${ }^{2}$, \\ Tadashi Honda ${ }^{3}$, Colin J Henderson ${ }^{1}$ and C Roland Wolf ${ }^{\star}, 1$ \\ ${ }^{1}$ Division of Cancer Research, School of Medicine, University of Dundee, Ninewells Hospital, Dundee DD1 9SY, UK; ${ }^{2}$ Department \\ of Pathology and Neuroscience, Ninewells Hospital and Medical School, Dundee DD1 9SY, UK and ${ }^{3}$ Department of Chemistry and \\ Institute of Chemical Biology and Drug Discovery, Stony Brook University, Stony Brook, NY 11794-3400, USA
}

Background: Although the nuclear factor-erythroid 2-related factor 2 (NRF2) pathway is one of the most frequently dysregulated in cancer, it is not clear whether mutational status is a good predictor of NRF2 activity. Here we utilise four members of the aldoketo reductase (AKR) superfamily as biomarkers to address this question.

\begin{abstract}
Methods: Twenty-three cell lines of diverse origin and NRF2-pathway mutational status were used to determine the relationship between AKR expression and NRF2 activity. AKR expression was evaluated in lung cancer biopsies and Cancer Genome Atlas (TCGA) and Oncomine data sets.
\end{abstract}

Results: AKRs were expressed at a high basal level in cell lines carrying mutations in the NRF2 pathway. In non-mutant cell lines, co-ordinate induction of AKRs was consistently observed following activation of NRF2. Immunohistochemical analysis of lung tumour biopsies and interrogation of TCGA data revealed that AKRs are enriched in both squamous cell carcinomas (SCCs) and adenocarcinomas that contain somatic alterations in the NRF2 pathway but, in the case of SCC, AKRs were also enriched in most other tumours.

Conclusions: An AKR biomarker panel can be used to determine NRF2 status in tumours. Hyperactivation of the NRF2 pathway is far more prevalent in lung SCC than previously predicted by genomic analyses.

NRF2 is a cap'n'collar (CNC) basic-region leucine zipper (bZIP) transcription factor that regulates a diverse battery of cytoprotective genes that collectively allow cells to survive transient periods of exposure to electrophilic, oxidative and inflammatory stress (Itoh et al, 1997; Hayes et al, 2010). The stability, abundance and activity of NRF2 is primarily governed by kelch-like ECH-associated protein 1 (KEAP1), a homodimeric substrate adaptor that recruits the factor to the Cullin 3/Ring-box 1 (CUL3/RBX1) E3 ubiquitin ligase holoenzyme, targeting it for ubiquitylation and subsequent proteasomal degradation (Itoh et al, 1999; McMahon et al, 2003). This targeting is inhibited by toxic electrophiles, such as lipid peroxidation products or the reactive metabolites of chemicals or therapeutic drugs, which directly modify reactive cysteine residues on KEAP1, leading to nuclear accumulation of NRF2 and increased transcription of cytoprotective genes (Dinkova-Kostova et al, 2002).

The increased capacity to inactivate genotoxic electrophiles conferred by activation of NRF2 can reduce the rate at which normal cells accumulate mutations, and can thereby inhibit chemically mediated carcinogenesis in animal models. Accordingly, Nrf2-null mice are more susceptible to this process (RamosGomez et al, 2001). However, following tumorigenesis, NRF2 may

*Correspondence: Professor CR Wolf; E-mail: c.r.wolf@dundee.ac.uk

${ }^{4}$ Current address: RECAMO, Masaryk Memorial Cancer Institute, Zluty kopec 7, 65653 Brno, Czech Republic

Received 27 July 2016; revised 7 October 2016; accepted 8 October 2016; published online 8 November 2016

(c) 2016 Cancer Research UK. All rights reserved 0007 - 0920/16

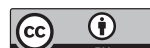


have a role in the malignant progression of lung adenoma to adenocarcinoma as the incidence and malignant characteristics of tumours in wild-type animals are greater than those in Nrf2knockout animals (Satoh et al, 2013). Generally, once a cancer has emerged, increased NRF2 activity is thought to promote cell survival and proliferation under conditions of environmental stress or in the face of chemotherapy (Hayes and McMahon, 2009). Consistent with this, genetic, epigenetic and signalling changes in tumours that exacerbate NRF2 activity have been associated with poor patient outcome (Shibata et al, 2008b). For this reason, NRF2 inhibition has emerged as a potential chemotherapeutic strategy.

Research into NRF2 has been impeded by the lack of sufficiently specific and sensitive antibodies. Its abundance is often, therefore, inferred from target gene/protein expression levels. This target gene battery is well defined in mouse, but is not so thoroughly characterised in man. Microarray and ChIP-Seq analyses of mouse cells have established that the CNC/bZIP factor is involved in the transcriptional regulation of several hundred genes (Thimmulappa et al, 2002; Malhotra et al, 2010). In human cell lines, a number of studies have identified the transcriptomic and, in some cases, proteomic changes that arise upon chemical or genetic perturbation of NRF2 signalling (MacLeod et al, 2009; Agyeman et al, 2012). Much of the NRF2 target gene battery is conserved between mouse, rat and man. Common targets include genes involved in antioxidant processes, NADPH generation, metal binding and the stress response (Hayes et al, 2010). There is divergence, however, in the drug metabolism enzyme targets of NRF2. In mouse, glutathione $S$-transferases (GSTs) predominate, while the aldoketo reductases (AKRs) are more conspicuous when human cells are examined. AKR1B10, AKR1C1, AKR1C2 and AKR1C3 constitute some of the most inducible targets of NRF2 in human systems, both normal and tumour-derived (Lou et al, 2006; MacLeod et al, 2009; Agyeman et al, 2012; Jung et al, 2013). The DNA motif bound by NRF2, the antioxidant response element (ARE), has been identified in the promoters of AKR1B10, AKR1C1 and AKR1C2 (Lou et al, 2006; Nishinaka et al, 2011).

In order to examine whether the ability of NRF2 to drive $A K R$ gene transcription is ubiquitous in human cell types of different origin, we have measured the expression of mRNA and protein for these targets in a panel of cultured cell lines (mostly tumourderived) in both basal and induced states. We show that AKR expression is related to the status of the NRF2/KEAP1 pathway and can be used as a readout for the activation or inhibition of NRF2. Through immunohistochemical analysis of lung tumour biopsies and interrogation of data from The Cancer Genome Atlas (TCGA), we have found that AKRs are enriched in both SCC and AC that contain somatic alterations in the NRF2 pathway but, in the case of SCC, AKR enrichment also occurs in cells not carrying NRF2 or KEAP1 mutation. These data indicate that NRF2 is frequently constitutively activated by alternative mechanisms in this cancer type and, therefore, that genetic analyses alone cannot determine the contribution of NRF2 to the tumour phenotype.

\section{MATERIALS AND METHODS}

Cell lines. A panel of cell lines containing either wild type or mutant forms of NRF2 or KEAP1, as confirmed by the Wellcome Trust Sanger Institute COSMIC database, was assembled. The origin, authentication and culture conditions of these cell lines are described in detail in Supplementary Materials and Methods. Characteristics of the mutant cell lines are detailed in Supplementary Table 1. All lines were free of mycoplasma contamination, as verified using the MycoAlert Mycoplasma detection kit (Lonza, Basel, Switzerland). In contrast to previous reports (Singh et al, 2006), the Wellcome Trust Sanger Institute
COSMIC database states that the $\mathrm{H} 23$ cell line contains a homozygous mutation in KEAP1 (579G $>$ C, Q193H), while neither H1395 nor H1993 contain the reported heterozygous mutation (both 1048G > A, G350S). Our sequencing of these cell lines agreed with the Sanger database entries in each case (Supplementary Figure 1).

Cell treatments, sample preparation and ELISA. Sulforaphane (SFN) was purchased from LKT laboratories (St Paul, MN, USA). ( \pm )- $(4 \mathrm{a} \alpha, 8 \mathrm{a} \alpha, 10 \mathrm{a} \beta)-1,2,4 \mathrm{a}, 6,8 \mathrm{a}, 9,10,10 \mathrm{a}-$ octahydro- $8 \mathrm{a}-$ ethynyl-1,1,4a-trimethyl-2,6-dioxophenanthrene-3,7-dicarbonitrile (TBE-31) was synthesised as described previously (Honda et al, 2007; Saito et al, 2013). Cells were treated with $5 \mu \mathrm{moll}^{-1} \mathrm{SFN}$ or $0.2 \mu \mathrm{moll}^{-1}$ TBE-31 in $0.1 \%$ acetonitrile vehicle on reaching $50-70 \%$ confluency, and protein or cDNA samples prepared $24 \mathrm{~h}$ later. For knockdown experiments, A549 and H838 cells were reverse-transfected with ON-TARGETplus NRF2 (L-003755-00) and non-targeting (D-001810-10-05) siRNA SMARTpools, each containing four siRNAs (Dharmacon, Thermo Fisher Scientific, Waltham, MA, USA), at a final concentration of $10 \mathrm{nmoll}^{-1}$, in complex with Lipofectamine RNAiMAX (Life Technologies, Carlsbad, CA, USA). Cells were lysed for analysis at the timepoints indicated. For fractionation of nuclear and cytoplasmic compartments, cells were processed using the NE-PER Nuclear and Cytoplasmic Extraction kit (Thermo Fisher Scientific). ELISAs were carried out by the In-Cell colorimetric method (Thermo Fisher Scientific) and statistical significance evaluated by unpaired $t$-test: ${ }^{\star} P \leqslant 0.05,{ }^{* *} P \leqslant 0.01,{ }^{* *} P \leqslant 0.001$.

Antibodies and western blotting. Rabbit polyclonal antiAKR1B10, anti-AKR1C1, anti-NQO1, anti-KEAP1 and anti-NRF2 were kindly provided by Professor John Hayes, University of Dundee. Mouse polyclonal anti-AKR1C1 (ab72576), mouse monoclonal antiGAPDH (ab8245), mouse monoclonal anti-TBP (ab51841) and rabbit monoclonal anti-LDH (ab52488) were purchased from Abcam (Cambridge, UK). Mouse monoclonal anti-AKR1C3 was a kind gift from Professor Trevor Penning, University of Pennsylvania, PA, USA. SDS-PAGE and western blotting were carried out as described previously (MacLeod et al, 2009).

Measurement of mRNA levels. mRNA analysis was carried out in 96-well plate format using Applied Biosystems' TaqMan Gene Expression Cells-to-CT kit (Life Technologies) according to the manufacturer's instructions. All real-time PCR primer and probe sets were purchased from Applied Biosystems (Foster City, CA, USA); AKR1B10 (Hs00252524_m1), AKR1C1/2 (Hs00413886 _m1), AKR1C3 (Hs00366267_m1), NRF2 (Hs00975960_m1), Actin B internal standard (4352935E). Assays were performed in triplicate and fold changes calculated using the $2(-\delta \delta \mathrm{C}(\mathrm{T}))$ method. Fold changes for inducer-treated cells were calculated relative to vehicle-treated cells and the heatmap generated using ggplot2 in R. Statistical significance was evaluated using an unpaired $t$-test. For Figures $3 \mathrm{~B}$ and $\mathrm{C}$ : ${ }^{\star} P \leqslant 0.05,{ }^{*} P \leqslant 0.01$, ${ }^{* * *} P \leqslant 0.001$. For Supplementary Table 2 , FDR $(\mathrm{Q})$ was set to $1 \%$ and significant differences are denoted ' '.

TCGA data retrieval and analysis. All gene expression and mutation data for AC and SCC were derived from publicly available TCGA datasets (15 July 2015, https://tcga-data.nci.nih.gov/tcga/). For AC, analyses were performed on the 'TCGA, Nature 2014' data set. For SCC, analyses were performed on the 'TCGA Provisional' data set (mutation information from samples shared with the 'TCGA, Nature 2012' data set). Normalised level 3 RNA-seq gene expression values, as determined using the RNASeq by Expectation Maximisation (RSEM) algorithm, were retrieved using the TCGA-Assembler package in R and RStudio (Zhu et al, 2014). Mutation data for NRF2 and KEAP1 were retrieved using the cBioPortal web application programming interface (www.cbioportal.org). For the comparison of wild type 
with mutant non-small cell lung cancer (NSCLC), we analysed 230 cases of AC (179 wild type, 51 mutant) and 178 cases of SCC (120 wild type, 58 mutant). For the comparison of tumour to matched normal tissue we analysed data for 45 tumour/normal (35 wild type, 10 mutant) paired $\mathrm{AC}$ and 16 tumour/normal (11 wild type, 5 mutant) paired SCC samples. All data processing and statistical analyses were carried out in $\mathrm{R}$ using packages cgdsr, plyr and ggplot2. Association was evaluated by unpaired and paired Wilcoxon ranked test for wild-type vs mutant values (Figure 4) and matched normal $v s$ tumour values (Figure 5), respectively.

\section{RESULTS}

NRF2 or KEAP1 mutation leads to a high basal level of AKR1B, AKR1C1/2 and AKR1C3 expression. Whole-cell lysates were prepared from a panel of cell lines, some of which carry mutations in NRF2 or KEAP1, and AKR protein expression measured by western blotting (Figure 1). The rabbit antibody raised against AKR1B10 (36.02 kDa) also reacts with AKR1B1 $(35.85 \mathrm{kDa})$. Similarly, as AKR1C1 $(36.79 \mathrm{kDa})$ and AKR1C2 $(36.74 \mathrm{kDa})$ proteins are $98 \%$ identical, it is likely that the mouse polyclonal antibody used to detect AKR1C1 cross-reacts with AKR1C2. The mouse monoclonal antibody used to detect AKR1C3 is highly specific; cross-reactivity with recombinant forms of human AKR1A, AKR1B, AKR1C and AKR1D superfamily members was not observed (data not shown).

AKR1B, AKR1C1/2 and AKR1C3 were all expressed constitutively at the highest levels in A549, H460 (both of which are homozygous for mutations in KEAP1) and HO1-u-1 (heterozygous for NRF2 mutation, Figure 1). In addition, AKR1C1/2 was also highly expressed in LK-2 cells (homozygous for NRF2 mutation), while AKR1C3 was expressed at low, but detectable, levels in H838 (homozygous for KEAP1 mutation), 5637 and MDA-MB-231 (KEAP1 and NRF2 wild type) cells (Figure 1). It should be noted that none of the cell lines possess mutant CUL3, with the exception of H460 (1299C > T, T410I), which also carries mutant KEAP1 (Supplementary Table 1).

AKR1B, AKR1C1/2 and AKR1C3 are inducible in cell lines with functional, but not mutated, KEAP1/NRF2. Cell lines were treated for $24 \mathrm{~h}$ with two NRF2 inducers; SFN, an isothiocyanate and a widely used activator of NRF2, and TBE-31, a highly potent

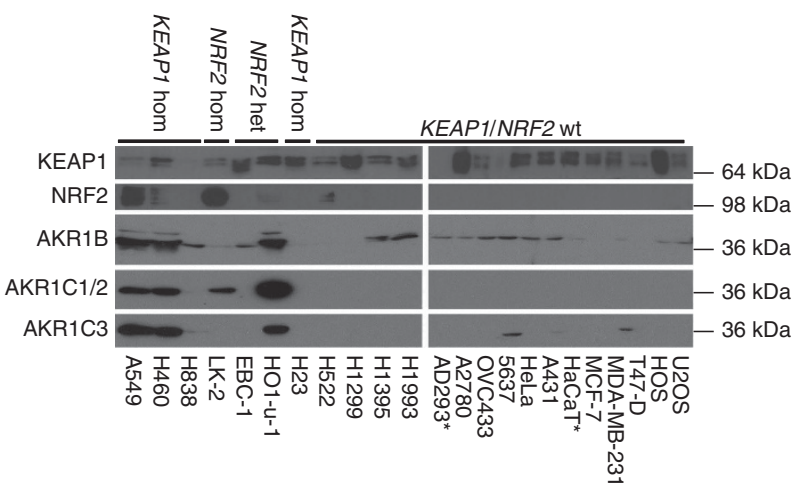

Figure 1. AKR1B, AKR1C1/2 and AKR1C3 are expressed at high levels in cell lines carrying mutant KEAP1 or NRF2. Western blotting of whole-cell lysates for AKR1B, AKR1C1/2 and AKR1C3 was carried out as described in Materials and Methods. Samples were prepared and analysed in parallel and uniformity of sample loading on the gels was verified by Coomassie blue staining (data not shown). ${ }^{*} N R F 2$ and KEAP1 mutational status in AD293 and HaCaT cell lines unknown. tricyclic-bis-enone inducer (Honda et al, 2007; Liby et al, 2008). Remarkably, Taqman analysis of RNA indicated that SFN and TBE-31 induced $A K R 1 B 10, A K R 1 C 1 / 2$ and $A K R 1 C 3$ mRNA in almost all tumour cell lines with wild-type KEAP1 and NRF2, independent of the tissue or origin (Figure 2, fold change values are provided in Supplementary Table 2). At the concentrations tested, fold induction in response to TBE-31 was generally much greater than in response to SFN. The presence of two cyano enone moieties within TBE-31 have previously been shown to underpin this high relative potency. With the exception of $\mathrm{H} 23$, AKRs were not inducible in cell lines with biallelic mutations in KEAP1 or NRF2. In one cell line carrying heterozygous NRF2 mutation, EBC-1, AKR mRNAs could still be modestly upregulated in response to inducer treatment.

In many of the cell lines homozygous for wild-type KEAP1 and $N R F 2$, the induction of $A K R$ mRNA was paralleled by an increase in protein (Figure $3 \mathrm{~A}$, summarised in Supplementary Table 3). In some cell lines, all targets were induced (for example, OVC433, A431, HaCaT and MCF-7 cells) while, in others the induction was much less marked. The western blots shown in Figure 3A were exposed for varying lengths of time in order to optimise chemiluminescent signal intensity to determine AKR induction on a cell line basis. The data should therefore not be compared directly with Figure 1. In many cases, AKR expression was close to or below the limit of detection with the blotting protocol applied, preventing a full comparison with the mRNA data. No induction of AKR proteins was detected in any of the KEAP1/NRF2 mutant cell lines, with the exception of AKR1C1/2 in HO1-u-1 cells. Western blotting for $\mathrm{NAD}(\mathrm{P}) \mathrm{H}$ dehydrogenase, quinone 1 (NQO1), the protein most commonly used as a proxy measure of NRF2 activity, showed that it was constitutively expressed in almost all of the cell lines, being undetectable in only AD293 and MDA-MB-231. NQO1 was inducible in many of the lines homozygous for wild-type KEAP1 and NRF2, but the fold changes were less than those of the AKRs. NQO1 was not inducible in the mutant cell lines.

Knockdown of NRF2 in KEAP1 homozygous mutant cells leads to decreases in AKR1C1 expression. The A549 and H838 cell lines, which lack functional KEAP1 and hence exhibit elevated NRF2 activity (Singh et al, 2006), were transfected with either nontargeting siRNA (siNT), or siRNA against NRF2 (siNRF2). In both cell lines, NRF2 knockdown reduced the mRNA level to between 20 and $50 \%$ of control level (Figure 3B), with a concomitant decrease in the level of AKR1C1/2 mRNA to $\sim 5-20 \%$ of control, after $48 \mathrm{~h}$ (Figure 3C). Protein levels for both NRF2 and AKR1C1/2 were decreased in A549 cells, and this effect was maintained up to $120 \mathrm{~h}$ (Figure 3D). AKR1C1/2 depletion after $120 \mathrm{~h}$ was also observed by the In-Cell ELISA method, using mouse polyclonal anti-AKR1C1, in both A559 and H838 cells (Figure 3E). The proportion of decrease in $\mathrm{AKR} 1 \mathrm{C} 1 / 2$ expression is similar to that of other NRF2 targets, as described in previous studies (Singh et al, 2006, 2008).

AKR1B, AKR1C1/2 and AKR1C3 are co-ordinately upregulated in the majority of lung SCC and in the minority of lung AC. Rabbit polyclonal anti-AKR1B10 and anti-AKR1C1 and mouse monoclonal anti-AKR1C3 were used for immunohistochemistry. All three of these primary antibody preparations give single bands on western blot of whole-cell lysate protein samples from A549 cells, indicating their specificity (Supplementary Figure 2). We initially determined whether the antibodies were suitable for immunohistochemistry by staining FFPE preparations of cultured cell lines; the MCF-7-derived AREc32 cell line, in which NRF2 activity is inducible, and the A549 cell line, in which NRF2 activity is high due to a homozygous inactivating mutation in KEAP1 (Singh et al, 2006). The anticipated pattern of expression was observed, wherein AREc32 cells show minimal staining that is 
increased following treatment with $5 \mu \mathrm{M}$ SFN for $24 \mathrm{~h}$, and A549 cells show the strongest staining, reflecting their KEAP1 mutant status (Supplementary Figure 3).

Fifteen FFPE samples of NSCLC were classified by conventional histological criteria as either SCC $(n=8)$ or AC $(n=7)$. Positive immunohistochemical staining was detected for at least one AKR in all SCC samples and for two or three AKRs in seven of eight samples (scoring provided in Table 1, representative images shown in Supplementary Figure 4). Staining was observed for all three AKRs in two of seven AC, while no staining was detected in the remaining five. The difference between SCC and AC was found to be statistically significant $(P=0.0002)$ by two-tailed Fisher's exact test.

AKR mRNA is highly enriched in KEAP1/NRF2 mutant AC and SCC, but also in KEAP1/NRF2 wild-type SCC. Data were retrieved from TCGA for NSCLC as described in Materials and Methods. A comparison of samples wild type or mutant for the NRF2 pathway demonstrated a highly significant enrichment of mRNA for all four $A K R$ genes in mutant cases, in both $A C$ and SCC (Figure 4). In each of the AKR1C1 and AKR1C2 plots, we noted what appeared to be a distinct cluster of mutant AC $(16 \%$ and $18 \%$ of the total mutant population, respectively) that had low expression of the transcript, approximately equal to the wild-type median level. Case IDs for the 10 samples with the lowest level of expression of each of the four genes under study were compared (Supplementary Table 4). There was a high degree of similarity between the lists indicating that if one of the transcripts was expressed at a low level it was likely that the other three were also expressed at a low level. These cases contained a variety of the specified mutational types: segregation to the low-expression cluster was not dependent on only one type of pathway alteration. Moreover, when $A K R$ data from SCC were assessed for coexpressed genes in cBioPortal (www.cbioportal.org), strong correlations were observed between $A K R$ s, and of $A K R$ s with other NRF2-regulated genes, such as GPX2, GSR, PGD, G6PD, GCLM, GCLC, ME1, SRXN1 and NQO1 (Supplementary Table 5). There was no association of CUL3 mutation status with $A K R$ mRNA level in either AC or SCC when all samples were considered (Supplementary Figure 5), although there was a statistically significant increase in $A K R 1 B 10, A K R 1 C 1$ and AKR1C2 mRNA in SCC when cases which were mutant for KEAP1 and/or NRF2 were removed from the wild-type population (Supplementary Figure 6).

We compared matched tumour/normal samples from 51 cases of AC and 53 cases of SCC. For AC, all four AKRs showed a wider range of expression in tumour tissue than in normal tissue (Figure 5). This spread of values was largely associated with higher $A K R$ levels in NRF2/KEAP1 mutant cases. Overall, the median level of $A K R 1 C 1, A K R 1 C 2$ and $A K R 1 C 3$ was decreased in tumour, relative to normal tissue but when only mutant cases were considered, all AKRs were significantly increased. Paired Wilcoxon ranked test determined $P$-values of tumour $v s$ normal gene expression for matched mutant AC samples $(n=10)$ as follows; AKR1B10: 0.002, AKR1C1: 0.002, AKR1C2: 0.004, AKR1C3: 0.002. In SCC, all four $A K R$ s were highly significantly enriched in tumour tissue, compared with normal, irrespective of mutational status, although mutant cases generally possessed a higher relative level of

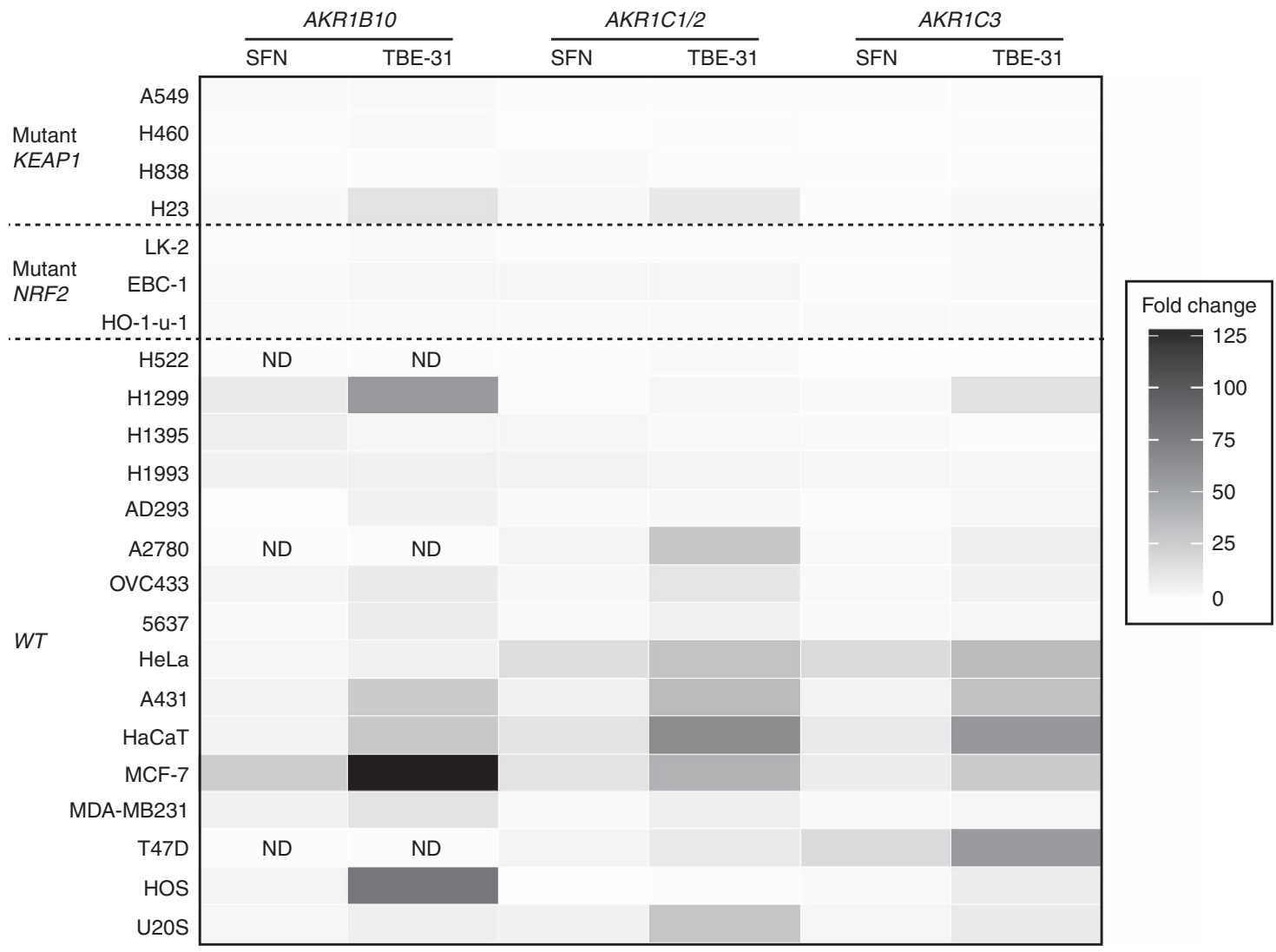

Figure 2. Chemical activators of NRF2 induce AKR mRNA in human cell lines with wild-type KEAP1 and NRF2. Cells were seeded, incubated under standard conditions for $24 \mathrm{~h}$, then treated with $5 \mu \mathrm{moll} \mathrm{I}^{-1} \mathrm{SFN}, 0.2 \mu \mathrm{moll} \mathrm{I}^{-1} \mathrm{TBE}-31 \mathrm{or}$ vehicle control. After a further $24 \mathrm{~h}$, cells were lysed, cDNA synthesised, and RT-PCR carried out for AKR1B10, AKR1C1/2 and AKR1C3. Fold change values for SFN and TBE-31-treated cells were calculated relative to vehicle control cells. ND: mRNA for AKR1B10 was not detected in H522, A2780 and T47D cells treated with vehicle, preventing accurate quantification of fold change. 
A
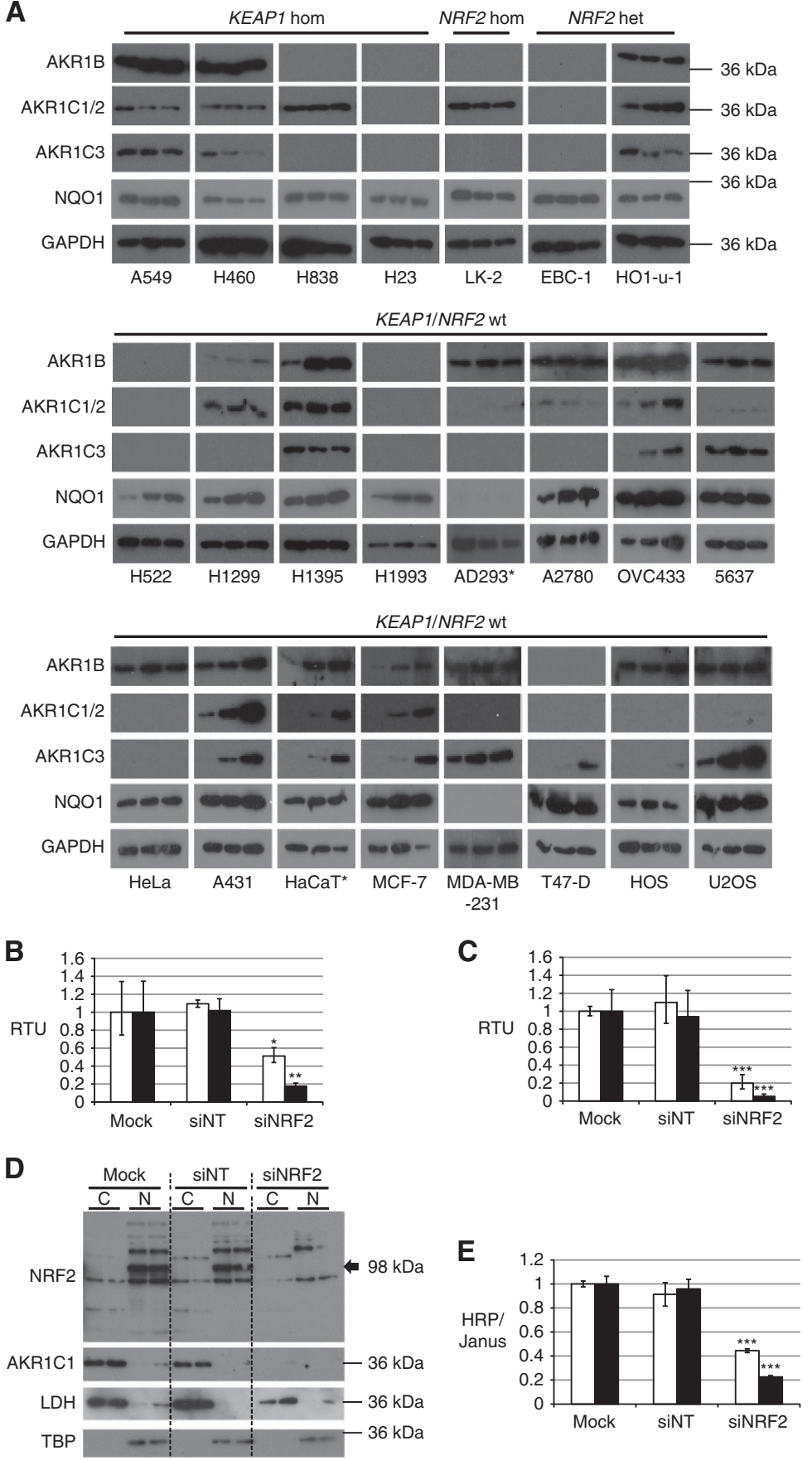

Figure 3. Constitutive and inducible expression of AKR proteins in human cell lines. (A) Chemical activators of NRF2 induce AKR protein in human cells possessing wild-type KEAP1/NRF2. Cells were seeded and incubated under standard conditions for $24 \mathrm{~h}$. Medium was replaced with fresh complete medium containing inducer compounds in acetonitrile (final concentration of acetonitrile, $0.1 \%(\mathrm{v} / \mathrm{v})$ ), then incubated for a further $24 \mathrm{~h}$. Protein samples were prepared from whole-cell lysates and immunoblotted for AKR1B, AKR1C1/2, AKR1C3 and NQO1. In each blot, lane 1 contains acetonitrile vehicle control, lane 2 contains $5 \mu \mathrm{moll} \mathrm{I}^{-1}$ SFN and lane 3 contains $0.2 \mu \mathrm{moll} \mathrm{I}^{-1}$ TBE-31. Data are representative of two separate experiments. ${ }^{*}$ NRF2 and KEAP1 mutational status in AD293 and HaCaT cell lines unknown. (B-E) Knockdown of NRF2 leads to decreased AKR1C1/2 expression in A549 and H838 cells. TaqMan RT-PCR was carried out for NRF2 (white bars) and AKR1C1 (black bars) in A549 cells (B) and H838 cells (C) $48 \mathrm{~h}$ after mock reverse transfection (transfection reagent alone), or reverse transfection with siRNA. RTU, relative transcription units. (D) Western blots were carried out for NRF2 (black arrow) and AKR1C1 in A549 cells $120 \mathrm{~h}$ after reverse transfection. Lactate dehydrogenase (LDH) and TATA binding protein (TBP) blots are shown to demonstrate effective separation of the cytoplasmic and nuclear fractions, respectively. (E) In-Cell ELISA of AKR1C1 was carried out $120 \mathrm{~h}$ after reverse transfection in A549 (white bars) and H838 (black bars) cells. HRP (horseradish peroxidase) signal was adjusted for cell density using Janus Green cell stain. For B, C and E, statistical significance was calculated relative to mock-transfected control cells. ${ }^{\star} P \leqslant 0.05,{ }^{\star} P \leqslant 0.01,{ }^{\star \star \star} P \leqslant 0.001$.

expression. Median fold enrichments in SCC were as follows: AKR1B10: 529-fold, AKR1C1: 16-fold, AKR1C2: 50-fold, AKR1C3: 7 -fold. This pattern of enrichment in AC and SCC was also seen with the other NRF2 target genes, GCLC, GCLM and NQO1, although HMOX1 was downregulated in both tumour types (Supplementary Figure 7). 
The association of $A K R 1 B 10$ mRNA expression with tumour type was investigated in separate microarray data sets using the Oncomine platform (www.oncomine.org). We found a highly significant association of $A K R 1 B 10$ overexpression with SCC of the

\section{Table 1. Immunohistochemical scoring for NSCLC biopsies}

\begin{tabular}{|l|c|c|c|}
\hline Case & AKR1B & AKR1C1/2 & AKR1C3 \\
\hline AC_1 & ++ & +++ & $+/-$ \\
AC_2 & - & - & - \\
AC_3 & - & - & - \\
AC_4 & - & - & - \\
AC_5 & - & - & - \\
AC_6 & - & + & + \\
AC_7 & $+/-$ & +++ & + \\
SCC_1 & + & - & ++ \\
SCC_2 & ++ & +++ & $+/-$ \\
SCC_3 & ++ & +++ & $+/-$ \\
SCC_4 & + & ++ & - \\
SCC_5 & $+/-$ & ++ & - \\
SCC_6 & - & + & - \\
SCC_7 & ++ & +++ & - \\
SCC_8 &
\end{tabular}

Abbreviations: $\mathrm{AC}=$ adenocarcinoma; $\mathrm{SCC}=$ squamous cell carcinoma. Intensity of $\mathrm{IHC}$ staining was scored in seven $\mathrm{AC}$ and eight SCC biopsies. - , none detected; + / - , trace; + , weak; ++ , moderate, +++ , strong. lung in multiple analyses; examples from Lee et al (2008) and Zhu et al (2010) are presented in Supplementary Figure 8. As with TCGA data, expression of AKR1B10 correlated with that of $A K R 1 C 1, A K R 1 C 2, A K R 1 C 3$ and other targets of NRF2, such as GPX2, GSR, PGD and G6PD, with widespread co-induction in SCC (examples from Lee et al (2008) and Zhu et al (2010) are shown in Supplementary Figure 9).

\section{DISCUSSION}

The NRF2 pathway may become activated in tumours through a large number of mechanisms. Core components of the pathway itself are some of the most frequently mutated genes in cancer, with mutations in NRF2 and KEAP1 occurring in diverse tumour types (Singh et al, 2006; Shibata et al, 2008a, b; Kim et al, 2010; Zhang et al, 2010; Yoo et al, 2012). NRF2 may be transcriptionally upregulated by oncogenic forms of K-Ras, B-Raf and Myc (Denicola et al, 2011), although it should be noted here that we did not find a correlation between mutation in these genes and enrichment for AKR transcripts (cBioPortal, data not shown). Alternatively, NRF2 activity may increase in response to stimuli of exogenous origin, such as chemotherapeutic drugs (McMahon et al, 2014) or dietary compounds. KEAP1 levels may be decreased
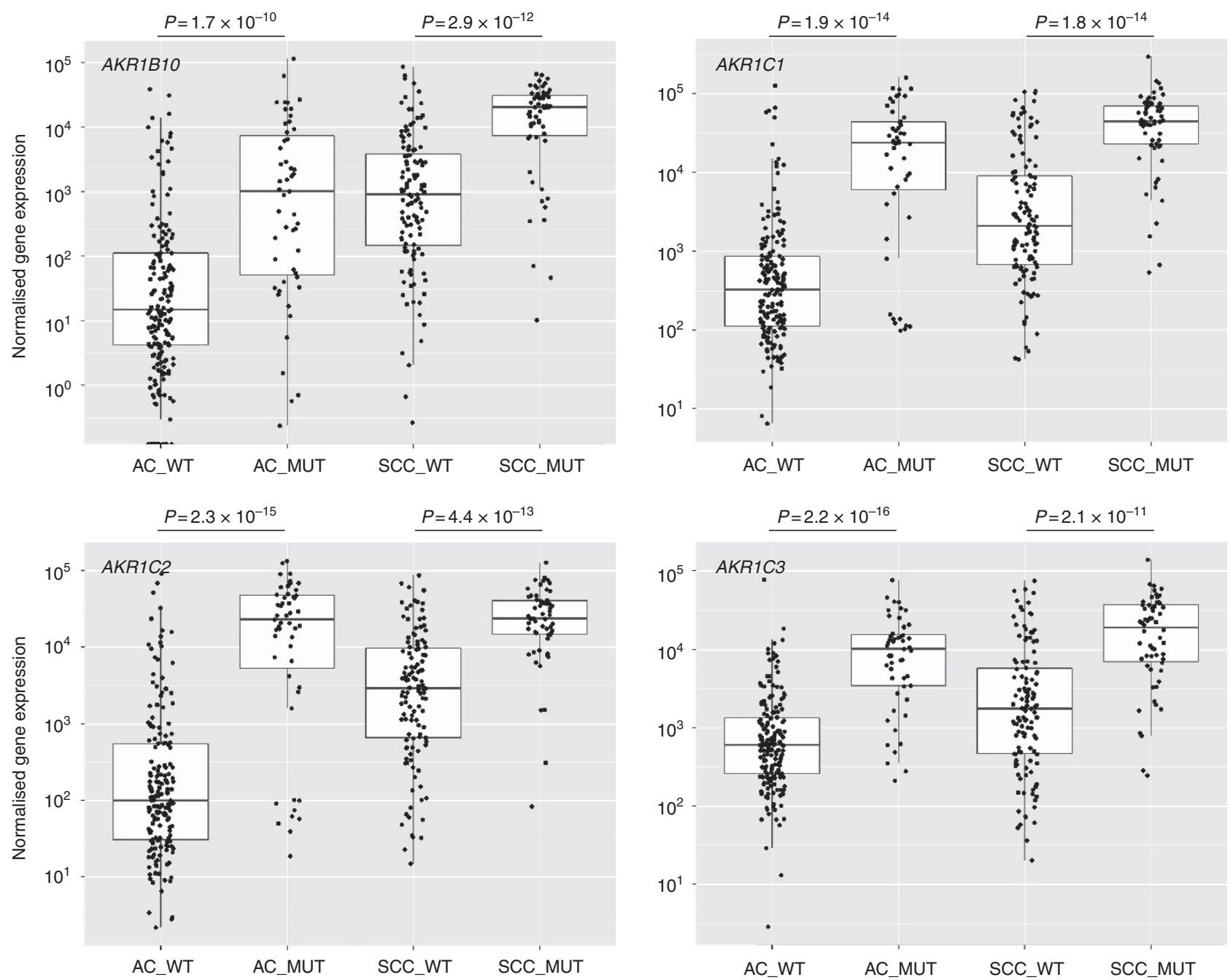

Figure 4. AKR mRNA is upregulated in both KEAP1/NRF2 mutant AC and SCC. TCGA data for NSCLC were processed and analysed as described in Materials and Methods. Both AC and SCC cases were defined as mutant (MUT) if they possessed one or more of the following: somatic mutation of KEAP1, loss of heterozygosity of KEAP1, somatic mutation of NRF2, gene amplification of NRF2. 

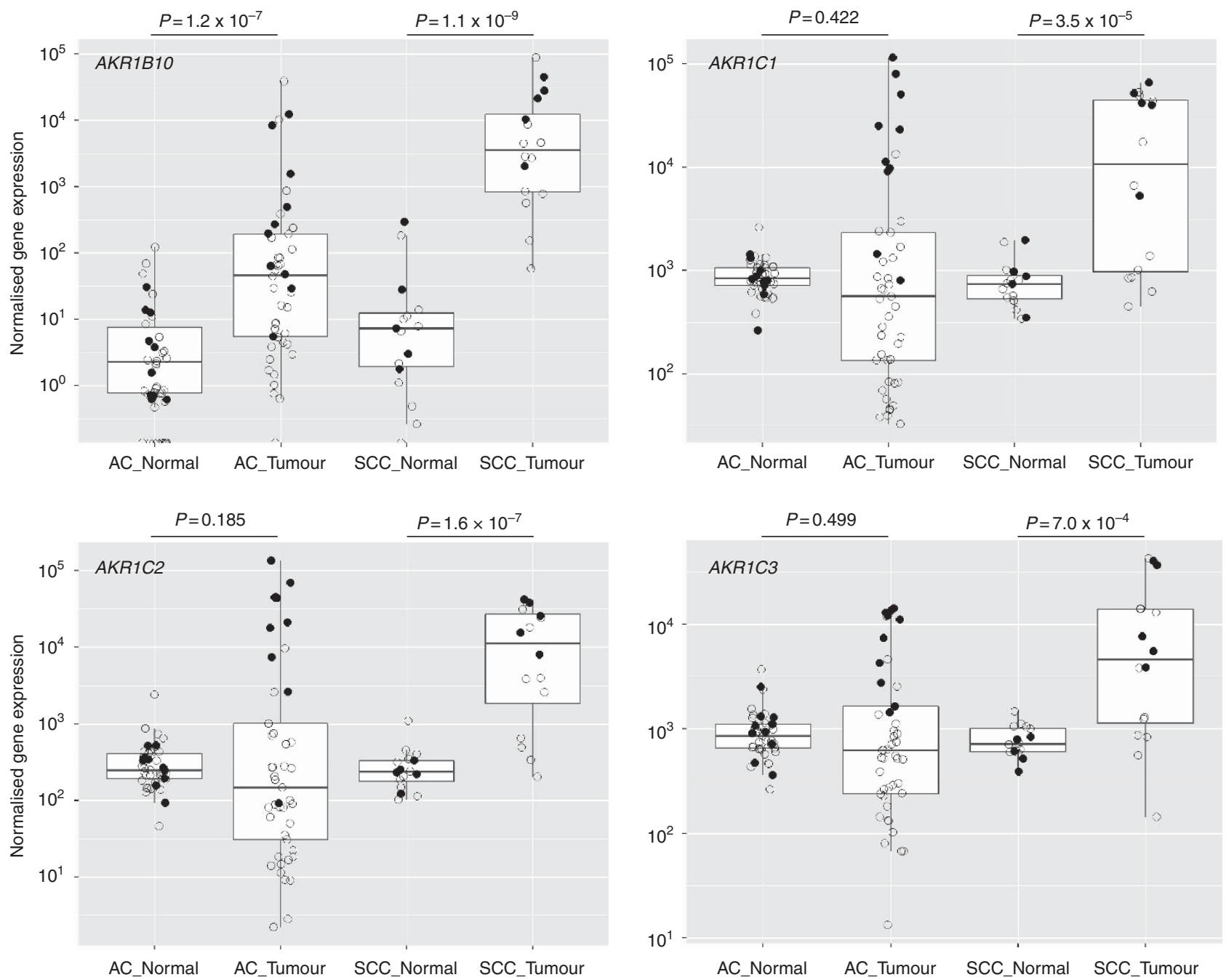

Figure 5. Induction of AKR mRNA is more widespread in SCC than in AC, irrespective of KEAP1/NRF2 mutation status. Paired normal/tumour sample data from TCGA were processed and analysed as described in Materials and Methods. Cases in which the tumour was either wild type (open circles) or mutant (closed circles) in respect to KEAP1/NRF2 mutation status are shown. Statistical significance of AKR enrichment relative to normal tissue is calculated as a combined score for both wild-type and mutant cases.

through promoter methylation (Martinez et al, 2013) or by microRNA-mediated interference (van Jaarsveld et al, 2013), or the protein itself may be chemically inactivated (Ooi et al, 2011). Mutation or deletion of CUL3, or methylation of its promoter, have been reported to occur at high frequencies in certain cancers (CGARN, 2012; Martinez et al, 2013). While CUL3 is undoubtedly a key regulator of NRF2 status, its interaction with a large number of substrate adaptor proteins facilitates the ubiquitination of many targets. Indeed, mutation of CUL3 did not correlate with increased expression of NRF2 target genes in the TCGA data sets, and actually tended towards co-occurrence with mutations in KEAP1 and NRF2 in SCC (cBioPortal, data not shown). We therefore disregarded this factor in our analyses to maximise specificity for the NRF2 pathway.

The implication of these (and other) observations is that constitutive NRF2 activity is far more common in tumours than has been predicted by the frequency of genomic or epigenomic alteration of the pathway, and that these analyses cannot therefore definitively predict its phenotypic status. In order to effectively interrogate CUL3/KEAP1/NRF2 pathway status, a robust downstream signature of activity is required. We had previously identified AKRs 1B10, 1C1, 1C2 and 1C3 as some of the most inducible targets of the human NRF2 pathway in the spontaneously immortalised keratinocyte cell line, HaCaT (MacLeod et al, 2009), a finding since replicated in nontumorigenic MCF10A and MCF12A breast epithelial cells, virally-immortalised HK-2 kidney cells and the U937 lymphoma cell line (Agyeman et al, 2012; Jung et al, 2013). Our aim in the present study was to determine the extent of this relationship across a large panel of cell lines and tumour samples.

We found that, generally, cultured cell lines are either responsive to inducers of NRF2, or have mutations in the pathway. In 23 cell lines of diverse origin we observed high constitutive levels of expression of AKR1B, AKR1C1/2 and AKR1C3 in three (A549, H460, HO1-u-1) of seven lines carrying mutant KEAP1 or NRF2. Further, two (H838, LK-2) of the remaining mutant lines exhibited high levels of one these enzymes individually. High levels of AKR coincided with high levels of NRF2. With very few exceptions (AKR1B in H1395 and H1993, and AKR1C3 in 5637 and MBA231), AKRs were expressed at relatively low constitutive levels in cell lines with non-mutated KEAP1/NRF2. Importantly, the true (relative) level of NRF2 activity in each of these cell lines is unknown. It has been shown, however, that certain KEAP1 mutations have little or no influence on the capacity of KEAP1 to repress NRF2 (Hast et al, 2014), which may explain why the H838 and $\mathrm{H} 23$ cell lines can carry mutant KEAP1 but express relatively 
low (compared with A549, H460 and HO-1-u-1) basal levels of AKR. In the case of H838, it has previously been shown that these cells exhibit lower levels of enzymatic activity of NQO1, the most commonly used proxy for NRF2, than do A549 and H460 (Singh et al, 2006). In the case of $\mathrm{H} 23$, these cells apparently retain the capacity for NRF2-mediated induction of AKRs, implying that the KEAP1/NRF2 axis is functional. Regarding the NRF2-mutant cell lines, and in agreement with our data, Shibata et al (2008b) reported that NRF2-driven luciferase activity in EBC1 cells was more responsive than that in LK2 cells to ectopic expression of KEAP1, suggesting that, as with KEAP1, mutations in NRF2 are not functionally equivalent. In our analysis of TCGA data, we observed a strong association of NRF2 pathway mutational status with $A K R$ mRNA levels, in agreement with the observations of Cescon et al (2015). Interestingly, we observed a subgroup of KEAP1/NRF2 mutant AC that did not show enrichment for $A K R$ mRNA. Conversely, we found that many cases of SCC in which KEAP1 and NRF2 were wild type exhibited higher levels of AKR expression than their matched normal controls. Importantly, these observations suggest that mutation in the KEAP1/NRF2 pathway and high levels of target gene expression are not interchangeable descriptors, and that a biomarker signature, such as that described here, provides a more definitive measure of NRF2 activation relative to a proxy measure such as genotype/epigenotype. Furthermore, the assessment of AKR levels potentially constitutes a means to discriminate between the relative functionality of KEAP1 and NRF2 mutations and, ultimately, whether they are likely to influence tumour phenotype.

As Phase I drug metabolism enzymes, AKRs have been implicated in the bioactivation and detoxification of chemotherapeutic drugs (Jin and Penning, 2007). Upregulation of AKR expression by NRF2 could therefore alter chemoresponse, with a potentially deleterious change in efficacy and/or toxicity. Overexpression of AKR1Cs has been identified as a mechanism of resistance to several commonly used agents, for example platinum compounds (Deng et al, 2004). AKRs have also been implicated in the genesis of cancer. Two distinct pro-proliferative functions have been identified for AKR1B10. First, this enzyme converts retinal to retinol, thereby preventing the generation of retinoic acid and consequently alleviating the anti-proliferative effect of this metabolite (Tang and Gudas, 2011). Second, AKR1B10 reduces farnesal and geranylgeranyl (Endo et al, 2009), a key step in the process of protein prenylation which is required by KRAS (and other oncoproteins) for their function. Consistent with this latter role, chemical inhibition of AKR1B10 was shown to inhibit carcinogenesis in a $\mathrm{Kras}^{\mathrm{G} 12 \mathrm{D}} / \operatorname{Trp} 53^{\mathrm{R} 172 \mathrm{H}}$ mouse model of pancreatic cancer, with an accompanying decrease in Ras signalling (Li et al, 2013). Moreover, this function may also, at least in part, explain why knockout of Nrf2 inhibits the genesis and proliferation of pancreatic and lung Kras ${ }^{\mathrm{G} 12 \mathrm{D}}$ tumours (Denicola et al, 2011). Of particular relevance to smokers, AKR enzymes metabolically activate polycyclic aromatic hydrocarbons, converting them to electrophilic and redox-active o-quinones (Palackal et al, 2002). These same enzymes are upregulated in response to cigarette smoke, indicating a further potential mechanistic contribution of NRF2 dysregulation to tumour genesis and promotion in this demographic (Zhang et al, 2008). Finally, and possibly relating to the hormonal metabolism functions of enzymes of the AKR1C subfamily, the enzymatic activity of ectopically expressed AKR1C1 and AKR1C2 has been shown to enhance tumourigenesis of NIH3T3 xenografts in mice (Chien et al, 2009). Collectively, these findings implicate AKRs in the genesis of cancer, extending their involvement beyond pathways of xenobiotic metabolism. This potential role is further evidenced by our demonstration of the ubiquitous nature of the NRF2/AKR relationship.

Upon challenge with chemical inducers of NRF2, we observed an increase in mRNA for $A K R 1 B 10, A K R 1 C 1 / 2$ and $A K R 1 C 3$ in almost all cell lines containing non-mutated KEAP1 and NRF2, whereas cell lines carrying heterozygous mutations in these genes were less responsive to these compounds and, with the exception of $\mathrm{H} 23$, homozygous mutant cell lines were completely nonresponsive. These findings strongly support the contention that AKRs are co-regulated as part of the NRF2 gene battery and, moreover, indicate their potential utility for inferring NRF2 hyperactivation in tumour tissue, irrespective of its mechanistic origin. Interestingly, AKRs were more markedly induced than NQO1 following chemical activation of NRF2 in wild-type cells, which is significant because NQO1 is currently the most widely used biomarker of NRF2 activation. This contrasts somewhat with the TCGA analysis, in which NQO1 performed similarly to AKRs. (Notably, HMOX1, another commonly used proxy for NRF2 levels, did not show any indication of differential expression in tumours, mutant or otherwise.) As all NRF2 target genes will be subject to multifactorial regulation, we suggest that the expression of AKRs should be considered a signature of NRF2 activity. This signature, while generally segregating with NRF2/KEAP1 genotype in cultured cells (i.e., higher expression in mutant cells), did not appear to do so in lung SCC, as high levels of AKR were detected in tumours without these mutations. This finding suggests that additional mechanisms of pathway regulation, potentially those with roots in the environmental or stromal context of the tumour, may often be key determinants of its status in vivo. In addition, our data support the contention that, while the transient activation of this pathway may be chemopreventive in normal cells, its chronic activation in cancer cells confers a selective advantage through enhanced proliferation and chemoresistance.

Non-small cell lung cancer is the tumour type in which KEAP1/ NRF2 dysfunction has most frequently been reported. Within NSCLC, mutations in KEAP1 occur primarily in AC and mutations in NRF2 occur primarily in SCC (Singh et al, 2006; Shibata et al, 2008b; Hayes and McMahon, 2009; Kim et al, 2010). More generally, NRF2 mutations are associated with squamous carcinomas irrespective of the tissue of origin. In one study of 1145 carcinoma samples from 13 tissue types, of which 167 were squamous, 21 of the 22 detected mutations in NRF2 were in SCC (Kim et al, 2010). In a separate integrative genomic and epigenomic analysis of SCC of the lung, somatic alteration of the CUL3/KEAP1/NRF2 pathway was detected in $34 \%$ of 178 cases (CGARN, 2012). Pertinently, most reports of AKR overexpression in cancer have involved NSCLC. Increased expression of AKR1B and AKR1C isoforms has been demonstrated to occur in both SCC and $\mathrm{AC}$ of the lung, but in agreement with our analyses these events are more common in the former subtype (Fukumoto et al, 2005; Inamura et al, 2005; Woenckhaus et al, 2006). Furthermore, our results are consistent with microarray measurements of AKR1B10 mRNA transcript levels in SCC and AC of the lung (Lee et al, 2008; Zhu et al, 2010). As AKR1B10 has been shown to be highly upregulated in other types of cancer (Balendiran et al, 2009), it would be interesting to determine whether, as with mutation of NRF2, increased AKR levels segregate to SCC of tissues other than lung. Future work could also incorporate other AKRs that are known to be regulated by NRF2 but have not been included in this study, such as AKR7A2 (Li et al, 2015).

The present work demonstrates that $A K R 1 B 10, A K R 1 C 1$, $A K R 1 C 2$ and $A K R 1 C 3$ are transcriptional targets strongly indicative of NRF2 status in human tumours, and that NRF2 is the upstream effector of AKR overexpression in cancer, particularly in tumours of squamous origin. Although a large number of studies have identified genetic, epigenetic, signalling and other changes that can lead to NRF2 hyperactivation in both AC and SCC of the lung, our data indicate that this is of more widespread downstream consequence in SCC and, moreover, this hyperactivation frequently occurs in the absence of any somatic mutation of the pathway. If, as is widely thought, activation of NRF2 supports 
tumour growth and proliferation, targeted inhibition of the NRF2 pathway may constitute a potential therapeutic avenue in most cases of this cancer type. In conclusion, therefore, the utilisation of AKRs as biomarkers of NRF2 status will ultimately help determine the significance of this pathway in cancer genesis and progression, and of its potential roles in disease management and the design of therapeutic strategies.

\section{ACKNOWLEDGEMENTS}

We thank Professor John Hayes, University of Dundee, UK, for rabbit polyclonal anti-AKR1B10, anti-AKR1C1 and anti-NQO1. We also thank Professor Trevor Penning, University of Pennsylvania, PA, USA, for mouse monoclonal anti-AKR1C3. We also thank the Tayside Tissue Bank for contributing to this study, and to Dr Andrew Cassidy at the Genetics Core Services Unit, Ninewells Hospital, Dundee, for DNA analysis. This work was supported by Cancer Research UK programme grant C4639/ A10822. PJC is supported by project MEYS-NPSI-LO1413. TH is supported by Reta Pharmaceuticals and Stony Brook Foundation.

\section{CONFLICT OF INTEREST}

The authors declare no conflict of interest.

\section{REFERENCES}

Agyeman AS, Chaerkady R, Shaw PG, Davidson NE, Visvanathan K, Pandey A, Kensler TW (2012) Transcriptomic and proteomic profiling of KEAP1 disrupted and sulforaphane-treated human breast epithelial cells reveals common expression profiles. Breast Cancer Res Treat 132: 175-187.

Balendiran GK, Martin HJ, El-Hawari Y, Maser E (2009) Cancer biomarker AKR1B10 and carbonyl metabolism. Chem Biol Interact 178: 134-137.

Cescon DW, She D, Sakashita S, Zhu CQ, Pintilie M, Shepherd FA, Tsao MS (2015) NRF2 pathway activation and adjuvant chemotherapy benefit in lung squamous cell carcinoma. Clin Cancer Res 21: 2499-2505.

CGARN (2012) Comprehensive genomic characterization of squamous cell lung cancers. Nature 489: 519-525.

Chien CW, Ho IC, Lee TC (2009) Induction of neoplastic transformation by ectopic expression of human aldo-keto reductase 1C isoforms in NIH3T3 cells. Carcinogenesis 30: 1813-1820.

Deng HB, Adikari M, Parekh HK, Simpkins H (2004) Ubiquitous induction of resistance to platinum drugs in human ovarian, cervical, germ-cell and lung carcinoma tumor cells overexpressing isoforms 1 and 2 of dihydrodiol dehydrogenase. Cancer Chemother Pharmacol 54: 301-307.

Denicola GM, Karreth FA, Humpton TJ, Gopinathan A, Wei C, Frese K, Mangal D, Yu KH, Yeo CJ, Calhoun ES, Scrimieri F, Winter JM, Hruban RH, Iacobuzio-Donahue C, Kern SE, Blair IA, Tuveson DA (2011) Oncogeneinduced Nrf2 transcription promotes ROS detoxification and tumorigenesis. Nature 475: 106-109.

Dinkova-Kostova AT, Holtzclaw WD, Cole RN, Itoh K, Wakabayashi N, Katoh Y, Yamamoto M, Talalay P (2002) Direct evidence that sulfhydryl groups of Keapl are the sensors regulating induction of phase 2 enzymes that protect against carcinogens and oxidants. Proc Natl Acad Sci USA 99: 11908-11913.

Endo S, Matsunaga T, Mamiya H, Ohta C, Soda M, Kitade Y, Tajima K, Zhao HT, El-Kabbani O, Hara A (2009) Kinetic studies of AKR1B10, human aldose reductase-like protein: endogenous substrates and inhibition by steroids. Arch Biochem Biophys 487: 1-9.

Fukumoto S, Yamauchi N, Moriguchi H, Hippo Y, Watanabe A, Shibahara J, Taniguchi H, Ishikawa S, Ito H, Yamamoto S, Iwanari H, Hironaka M, Ishikawa Y, Niki T, Sohara Y, Kodama T, Nishimura M, Fukayama M, Dosaka-Akita H, Aburatani H (2005) Overexpression of the aldo-keto reductase family protein AKR1B10 is highly correlated with smokers' nonsmall cell lung carcinomas. Clin Cancer Res 11: 1776-1785.
Hast BE, Cloer EW, Goldfarb D, Li H, Siesser PF, Yan F, Walter V, Zheng N, Hayes DN, Major MB (2014) Cancer-derived mutations in KEAP1 impair NRF2 degradation but not ubiquitination. Cancer Res 74: 808-817.

Hayes JD, McMahon M (2009) NRF2 and KEAP1 mutations: permanent activation of an adaptive response in cancer. Trends Biochem Sci 34: 176-188.

Hayes JD, McMahon M, Chowdhry S, Dinkova-Kostova AT (2010) Cancer chemoprevention mechanisms mediated through the Keap1-Nrf2 pathway. Antioxid Redox Signal 13: 1713-1748.

Honda T, Sundararajan C, Yoshizawa H, Su X, Honda Y, Liby KT, Sporn MB, Gribble GW (2007) Novel tricyclic compounds having acetylene groups at $\mathrm{C}-8 \mathrm{a}$ and cyano enones in rings $\mathrm{A}$ and $\mathrm{C}$ : highly potent anti-inflammatory and cytoprotective agents. J Med Chem 50: 1731-1734.

Inamura K, Fujiwara T, Hoshida Y, Isagawa T, Jones MH, Virtanen C, Shimane M, Satoh Y, Okumura S, Nakagawa K, Tsuchiya E, Ishikawa S, Aburatani H, Nomura H, Ishikawa Y (2005) Two subclasses of lung squamous cell carcinoma with different gene expression profiles and prognosis identified by hierarchical clustering and non-negative matrix factorization. Oncogene 24: 7105-7113.

Itoh K, Chiba T, Takahashi S, Ishii T, Igarashi K, Katoh Y, Oyake T, Hayashi N, Satoh K, Hatayama I, Yamamoto M, Nabeshima Y (1997) An Nrf2/small Maf heterodimer mediates the induction of phase II detoxifying enzyme genes through antioxidant response elements. Biochem Biophys Res Commun 236: 313-322.

Itoh K, Wakabayashi N, Katoh Y, Ishii T, Igarashi K, Engel JD, Yamamoto M (1999) Keap1 represses nuclear activation of antioxidant responsive elements by Nrf2 through binding to the amino-terminal Neh2 domain. Genes Dev 13: 76-86.

Jin Y, Penning TM (2007) Aldo-keto reductases and bioactivation/ detoxication. Апnu Rev Pharmacol Toxicol 47: 263-292.

Jung KA, Choi BH, Nam CW, Song M, Kim ST, Lee JY, Kwak MK (2013) Identification of aldo-keto reductases as NRF2-target marker genes in human cells. Toxicol Lett 218: 39-49.

Kim YR, Oh JE, Kim MS, Kang MR, Park SW, Han JY, Eom HS, Yoo NJ, Lee SH (2010) Oncogenic NRF2 mutations in squamous cell carcinomas of oesophagus and skin. J Pathol 220: 446-451.

Lee ES, Son DS, Kim SH, Lee J, Jo J, Han J, Kim H, Lee HJ, Choi HY, Jung Y, Park M, Lim YS, Kim K, Shim Y, Kim BC, Lee K, Huh N, Ko C, Park K, Lee JW, Choi YS, Kim J (2008) Prediction of recurrence-free survival in postoperative non-small cell lung cancer patients by using an integrated model of clinical information and gene expression. Clin Cancer Res 14: 7397-7404.

Li D, Ma S, Ellis EM (2015) Nrf2-mediated adaptive response to methyl glyoxal in HepG2 cells involves the induction of AKR7A2. Chem Biol Interact 234: 366-371.

Li H, Yang AL, Chung YT, Zhang W, Liao J, Yang GY (2013) Sulindac inhibits pancreatic carcinogenesis in LSL-KrasG12D-LSL-Trp53R172H-Pdx-1-Cre mice via suppressing aldo-keto reductase family 1B10 (AKR1B10). Carcinogenesis 34: 2090-2098.

Liby K, Yore MM, Roebuck BD, Baumgartner KJ, Honda T, Sundararajan C, Yoshizawa H, Gribble GW, Williams CR, Risingsong R, Royce DB, Dinkova-Kostova AT, Stephenson KK, Egner PA, Yates MS, Groopman JD, Kensler TW, Sporn MB (2008) A novel acetylenic tricyclic bis-(cyano enone) potently induces phase 2 cytoprotective pathways and blocks liver carcinogenesis induced by aflatoxin. Cancer Res 68: 6727-6733.

Lou H, Du S, Ji Q, Stolz A (2006) Induction of AKR1C2 by phase II inducers: identification of a distal consensus antioxidant response element regulated by NRF2. Mol Pharmacol 69: 1662-1672.

MacLeod AK, McMahon M, Plummer SM, Higgins LG, Penning TM, Igarashi K, Hayes JD (2009) Characterization of the cancer chemopreventive NRF2dependent gene battery in human keratinocytes: demonstration that the KEAP1-NRF2 pathway, and not the BACH1-NRF2 pathway, controls cytoprotection against electrophiles as well as redox-cycling compounds. Carcinogenesis 30: 1571-1580.

Malhotra D, Portales-Casamar E, Singh A, Srivastava S, Arenillas D, Happel C, Shyr C, Wakabayashi N, Kensler TW, Wasserman WW, Biswal S (2010) Global mapping of binding sites for Nrf2 identifies novel targets in cell survival response through ChIP-Seq profiling and network analysis. Nucleic Acids Res 38: 5718-5734.

Martinez VD, Vucic EA, Pikor LA, Thu KL, Hubaux R, Lam WL (2013) Frequent concerted genetic mechanisms disrupt multiple components of the NRF2 inhibitor KEAP1/CUL3/RBX1 E3-ubiquitin ligase complex in thyroid cancer. Mol Cancer 12: 124. 
McMahon M, Campbell KH, MacLeod AK, McLaughlin LA, Henderson CJ, Wolf CR (2014) HDAC inhibitors increase NRF2-signaling in tumour cells and blunt the efficacy of co-adminstered cytotoxic agents. PLoS One 9: e114055.

McMahon M, Itoh K, Yamamoto M, Hayes JD (2003) Keap1-dependent proteasomal degradation of transcription factor Nrf2 contributes to the negative regulation of antioxidant response element-driven gene expression. J Biol Chem 278: 21592-21600.

Nishinaka T, Miura T, Okumura M, Nakao F, Nakamura H, Terada T (2011) Regulation of aldo-keto reductase AKR1B10 gene expression: Involvement of transcription factor Nrf2. Chem Biol Interact 191: 185-191.

Ooi A, Wong JC, Petillo D, Roossien D, Perrier-Trudova V, Whitten D, Min BW, Tan MH, Zhang Z, Yang XJ, Zhou M, Gardie B, Molinie V, Richard S, Tan PH, Teh BT, Furge KA (2011) An antioxidant response phenotype shared between hereditary and sporadic type 2 papillary renal cell carcinoma. Cancer Cell 20: 511-523.

Palackal NT, Lee SH, Harvey RG, Blair IA, Penning TM (2002) Activation of polycyclic aromatic hydrocarbon trans-dihydrodiol proximate carcinogens by human aldo-keto reductase (AKR1C) enzymes and their functional overexpression in human lung carcinoma (A549) cells. J Biol Chem 277: 24799-24808.

Ramos-Gomez M, Kwak MK, Dolan PM, Itoh K, Yamamoto M, Talalay P, Kensler TW (2001) Sensitivity to carcinogenesis is increased and chemoprotective efficacy of enzyme inducers is lost in nrf2 transcription factor-deficient mice. Proc Natl Acad Sci USA 98: 3410-3415.

Saito A, Zheng S, Takahashi M, Li W, Ojima I, Honda T (2013) An improved synthesis of a hydroxymethyl tricyclic ketone from cyclohexanone, the key processes for the synthesis of a highly potent anti-inflammatory and cytoprotective agent. Synthesis (Mass) 45: 3251-3254.

Satoh H, Moriguchi T, Takai J, Ebina M, Yamamoto M (2013) Nrf2 prevents initiation but accelerates progression through the Kras signaling pathway during lung carcinogenesis. Cancer Res 73: 4158-4168.

Shibata T, Kokubu A, Gotoh M, Ojima H, Ohta T, Yamamoto M, Hirohashi S (2008a) Genetic alteration of Keap1 confers constitutive Nrf2 activation and resistance to chemotherapy in gallbladder cancer. Gastroenterology 135: 1358-1368, (e1 - 4).

Shibata T, Ohta T, Tong KI, Kokubu A, Odogawa R, Tsuta K, Asamura H, Yamamoto M, Hirohashi S (2008b) Cancer related mutations in NRF2 impair its recognition by Keap1-Cul3 E3 ligase and promote malignancy. Proc Natl Acad Sci USA 105: 13568-13573.

Singh A, Boldin-Adamsky S, Thimmulappa RK, Rath SK, Ashush H, Coulter J, Blackford A, Goodman SN, Bunz F, Watson WH, Gabrielson E, Feinstein E, Biswal S (2008) RNAi-mediated silencing of nuclear factor erythroid-2-related factor 2 gene expression in non-small cell lung cancer inhibits tumor growth and increases efficacy of chemotherapy. Cancer Res 68: 7975-7984.
Singh A, Misra V, Thimmulappa RK, Lee H, Ames S, Hoque MO, Herman JG, Baylin SB, Sidransky D, Gabrielson E, Brock MV, Biswal S (2006) Dysfunctional KEAP1-NRF2 interaction in non-small-cell lung cancer. PLoS Med 3: e420.

Tang XH, Gudas LJ (2011) Retinoids, retinoic acid receptors, and cancer. Annu Rev Pathol 6: 345-364.

Thimmulappa RK, Mai KH, Srisuma S, Kensler TW, Yamamoto M, Biswal S (2002) Identification of Nrf2-regulated genes induced by the chemopreventive agent sulforaphane by oligonucleotide microarray. Cancer Res 62: 5196-5203.

van Jaarsveld MT, Helleman J, Boersma AW, van Kuijk PF, van Ijcken WF, Despierre E, Vergote I, Mathijssen RH, Berns EM, Verweij J, Pothof J, Wiemer EA (2013) miR-141 regulates KEAP1 and modulates cisplatin sensitivity in ovarian cancer cells. Oncogene 32: 4284-4293.

Woenckhaus M, Klein-Hitpass L, Grepmeier U, Merk J, Pfeifer M, Wild P, Bettstetter M, Wuensch P, Blaszyk H, Hartmann A, Hofstaedter F, Dietmaier W (2006) Smoking and cancer-related gene expression in bronchial epithelium and non-small-cell lung cancers. J Pathol 210: 192-204.

Yoo NJ, Kim HR, Kim YR, An CH, Lee SH (2012) Somatic mutations of the KEAP1 gene in common solid cancers. Histopathology $\mathbf{6 0}$ : 943-952.

Zhang L, Lee JJ, Tang H, Fan YH, Xiao L, Ren H, Kurie J, Morice RC, Hong WK, Mao L (2008) Impact of smoking cessation on global gene expression in the bronchial epithelium of chronic smokers. Cancer Prev Res (Phila) 1: $112-118$.

Zhang P, Singh A, Yegnasubramanian S, Esopi D, Kombairaju P, Bodas M, Wu H, Bova SG, Biswal S (2010) Loss of Kelch-like ECH-associated protein 1 function in prostate cancer cells causes chemoresistance and radioresistance and promotes tumor growth. Mol Cancer Ther 9: 336-346.

Zhu CQ, Ding K, Strumpf D, Weir BA, Meyerson M, Pennell N, Thomas RK, Naoki K, Ladd-Acosta C, Liu N, Pintilie M, Der S, Seymour L, Jurisica I, Shepherd FA, Tsao MS (2010) Prognostic and predictive gene signature for adjuvant chemotherapy in resected non-small-cell lung cancer. J Clin Oncol 28: 4417-4424.

Zhu Y, Qiu P, Ji Y (2014) TCGA-assembler: open-source software for retrieving and processing TCGA data. Nat Methods 11: 599-600.

Supplementary Information accompanies this paper on British Journal of Cancer website (http://www.nature.com/bjc) 